\title{
The role of previous falls in major osteoporotic fracture prediction in conjunction with FRAX in older Chinese men and women: the Mr. OS and Ms. OS cohort study in Hong Kong
}

\author{
Y. Su $^{1}$ (D) J. Leung ${ }^{2} \cdot$ T. Kwok $^{1,2}$
}

Received: 30 November 2017 / Accepted: 26 December 2017 / Published online: 12 April 2018

(C) International Osteoporosis Foundation and National Osteoporosis Foundation 2018

\begin{abstract}
Falls are a major concern in terms of fracture risk. Although awareness rising for the absence of falls in the FRAX algorithm, our study only identified the independent predictive role of previous recurrent falls and their better conjunction use with FRAX for major osteoporotic fracture prediction in older Chinese men.
\end{abstract}

Keywords Falls $\cdot$ FRAX $\cdot$ Major osteoporotic fractures $\cdot$ Risk prediction

This study is the first to show the important relationship between previous falls and the future fracture risk, taking competing death into account, in old Chinese people. Moreover, the development of fall-adjusted FRAX score also shows the possibility or impossibility for the use of falls together with FRAX in clinical practice in older Chinese people. Our findings may provide some evidence about the role of previous falls in older people and their relationship with FRAX.

\section{Compliance with ethical standards}

Conflicts of interest None.

Electronic supplementary material The online version of this article (https://doi.org/10.1007/s00198-017-4373-9) contains supplementary material, which is available to authorized users.

\footnotetext{
T. Kwok

tkwok@cuhk.edu.hk

1 Department of Medicine and Therapeutics, Prince of Wales Hospital, The Chinese University of Hong Kong, Shatin, Hong Kong, China

2 Jockey Club Centre for Osteoporosis Care and Control, The Chinese University of Hong Kong, Shatin, Hong Kong, China
} 